\title{
¿Los nanomateriales pueden causar neurotoxicidad?
}

\section{Can nanomaterials cause neurotoxicity?}

\author{
Natalie Jiménez-Barrios, ${ }^{*, 1}$ Yolanda I. Chirino ${ }^{*, 2}$
}

\begin{abstract}
In this article, we explain: a) what nano-materials are; b) how we are exposed to them; c) what their applications are; d) how they could cause damage if they are deposited in the brain, and, e) what are the supporting evidences that could cause hurt. Finally, we suggest some considerations for the design and study of the effects of nanomaterials in the central nervous system. The use of manufactured nanomaterials that are used in the medical, robotics, electronics, mechanics and food industries, among many others, are composed of nanoparticles (from 1 to $100 \mathrm{~nm}$ in diameter) that can be deposited in the respiratory tract and be transferred to the bloodstream, subsequently, they are deposited in different tissues, which is worrisome because of the risk of crossing the blood-brain barrier and being deposited in the brain. In addition, many nanoparticles do not degrade, so it is unknown how long they could remain in the penetrating tissues. It is important to design studies that allow knowing the consequences of being exposed to nanomaterials not only in health conditions, but in the presence of preexisting pathologies including neurodegenerative diseases in order to determine if the exposure could exacerbate said disease and to what extent.
\end{abstract}

KEYWORDS: nanomaterial, nervous system, toxicity, nanoparticle, neurotoxicity.

RESUMEN: En este artículo, explicamos: a) qué son los nanomateriales; b) cómo estamos expuestos a ellos; c) cuáles son sus aplicaciones; d) cómo podrían causar daño si se depositan en cerebro, y, e) cuáles son las evidencias en que se sustentaría una posible causa de daño. Finalmente, sugerimos algunas consideraciones para el diseño y estudio de los efectos de nanomateriales en el sistema nervioso central. El uso de nanomateriales manufacturados que se emplean en el área médica, robótica, electrónica, mecánica y alimenticia, entre muchas otras, están compuestos por nanopartículas (de 1 a $100 \mathrm{~nm}$ de diámetro) que pueden depositarse en el tracto respiratorio y trasladarse al torrente sanguíneo y, posteriormente, ser depositados en diferentes tejidos, lo cual es preocupante por el riesgo de cruzar la barrera hematoencefálica y ser depositadas en el cerebro. Además, muchas nanopartículas no se degradan, por lo que se desconoce cuánto tiempo podrían permanecer en los tejidos que penetran. Es importante diseñar estudios que permitan conocer cuáles serían las consecuencias de estar expuesto a nanomateriales no sólo en condiciones de salud, sino en presencia de patologías preexistentes incluyendo enfermedades neurodegenerativas con el objetivo de determinar si la exposición podría exacerbar dicha enfermedad y en qué medida.

PALABRAS CLAVE: nanomaterial, sistema nervioso, toxicidad, nanopartícula, neurotoxicidad.

Recibido: 20 de julio de 2017. Aceptado: 30 de octubre de 2017.

* Universidad Nacional Autónoma de México, Facultad de Estudios Superiores-Iztacala, Unidad de Biomedicina, Laboratorio de Carcinogénesis y Toxicología. Estado de México, México.

${ }^{1}$ Estudiante de la licenciatura en Biología.

${ }^{2}$ Autora para correspondencia: (chirino@campus.iztacala.unam.mx). 


\section{¿Qué son los nanomateriales?}

Hoy en día es común escuchar acerca de la nanotecnología como un gran avance y con ello el papel esencial logrado en aspectos sociales, económicos, industriales, comerciales, médicos y, actualmente, en la vida cotidiana.

La base de esta tecnología es la elaboración y el empleo de nanomateriales, es decir, la materia a escala nanométrica. Los nanomateriales están compuestos de una gran cantidad de partículas, llamadas nanopartículas, que se encuentran en un intervalo de 1 a 100 nanómetros de diámetro (Unión Europea, 2011). Un nanómetro ( $\mathrm{nm}$ ) es 0.000000001 metros, como comparación podemos mencionar que el ancho del material genético (DNA) de cada célula de tu cuerpo mide tan solo $2 \mathrm{~nm}$ (figura 1).

Las aplicaciones de estos nanomateriales son indispensables en diferentes campos como la industria textil, farmacéutica, alimenticia, armamentística, robótica, informática, electrónica, mecánica y en la medicina, por mencionar algunos. Por ejemplo, los nanocompositos se utilizan en la industria alimenticia en la fabricación de empaques pues protegen los alimentos y son biodegradables, una alternativa para el uso de plásticos (Thiruvengadam et al., 2018).

FIGURA 1. Un nanomaterial está compuesto de nanopartículas que tienen entre 1 y 100 nanómetros de tamaño en alguna de sus dimensiones. Para comparar, el ancho del DNA es de aproximadamente 2 nanómetros $(\mathrm{nm})$.

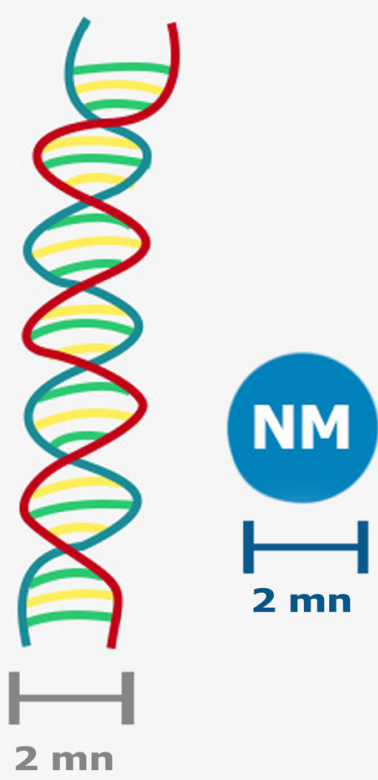

Fuente: Elaboración de las autoras.

\section{¿Cómo estamos expuestos a los nanomateriales?}

A nivel mundial, la producción de nanomateriales se encuentra en constante aumento, exponiendo a los trabajadores relacionados con su producción así como a la población a un mayor contacto con este tipo de materiales. Entre los principales nanomateriales a los que se encuentran expuestos están: a) los nanotubos de carbón; b) los nanocompositos; c) los fulerenos; d) los dendrímeros; e) las nanopartículas de base metálica como plata $(\mathrm{Ag})$, oro $(\mathrm{Au}) ; \mathrm{f})$ de base metálica o metaloide como los óxidos de hierro (Fe), dióxido de titanio $\left(\mathrm{TiO}_{2}\right)$, dióxido de estaño $\left(\mathrm{SnO}_{2}\right)$, óxido de aluminio $\left(\mathrm{Al}_{2} \mathrm{O}_{3}\right)$ y óxido de cerio $\left(\mathrm{CeO}_{2}\right)$, y dióxido de silicio $\left.\left(\mathrm{SiO}_{2}\right), \mathrm{y}, \mathrm{g}\right)$ quantum dots: compuestos de un núcleo de metales y una cubierta de composición diferente (figura 2). 
FIGURA 2. Clasificación de nanomateriales de acuerdo a su composición química. Au: oro; Ag: plata; $\mathrm{SiO}_{2}$, dióxido de silicio; $\mathrm{TiO}_{2}$, dióxido de titanio; $\mathrm{SnO}_{2}$, dióxido de estaño; $\mathrm{CdSe}$, selenurio de cadmio.

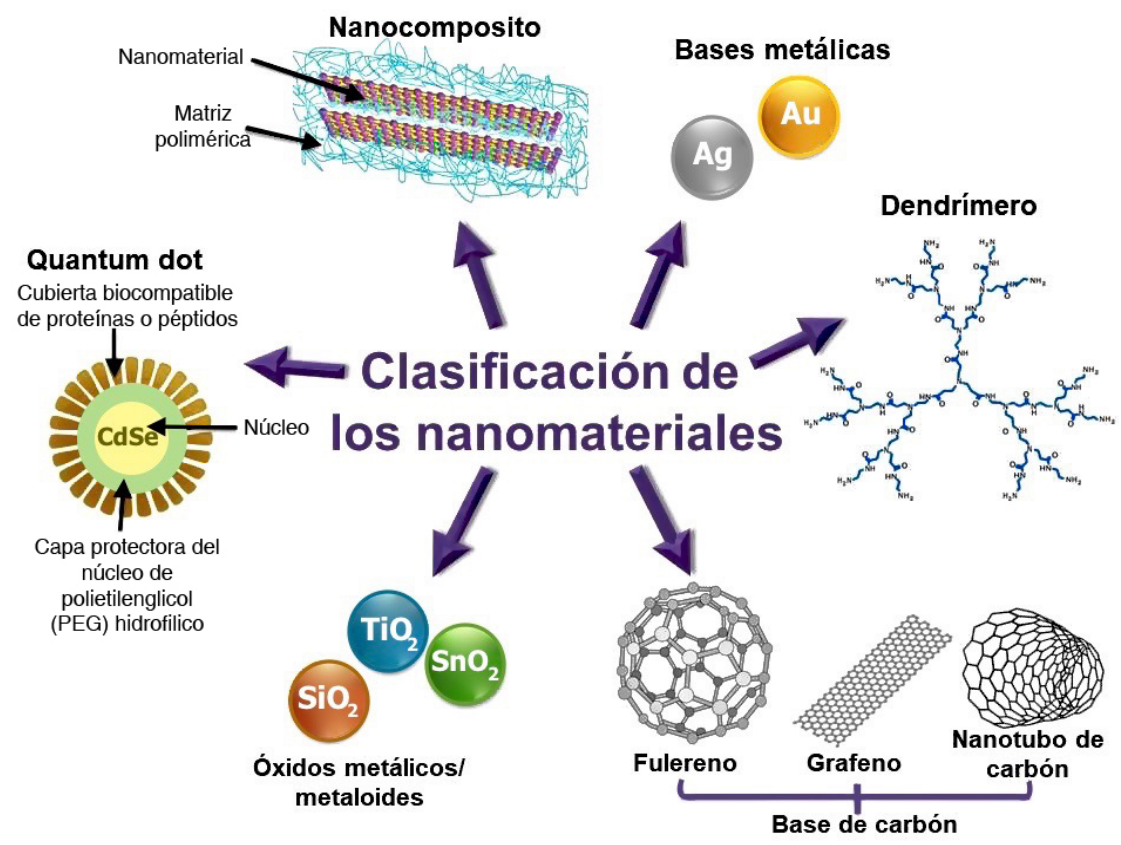

Fuente: Elaboración de las autoras.

Las nanopartículas que componen estos nanomateriales pueden entrar al cuerpo por la vía inhalatoria cuando son respiradas por personas en contacto con ellas en lugares donde se manufacturan. A estos lugares se les conoce como ambientes ocupacionales y en ellos los trabajadores pueden respirarlas durante sus jornadas de trabajo. También el personal de limpieza puede estar en contacto con estas partículas, pues una gran mayoría se fabrica en forma de polvos.

Los nanomateriales también pueden entrar al cuerpo a través de la vía oral cuando son consumidos en alimentos, ya sean sólidos o líquidos. Por ejemplo, en bebidas endulzadas, gomas de mascar o productos confitados. Algunos medicamentos pueden contener nanomateriales en forma de excipientes para tabletas.

El contacto con nanomateriales por la vía dérmica ocurre cuando son usados en cosméticos, por ejemplo, en bloqueadores solares o polvos cosméticos y en los ambientes ocupacionales, cuando las nanopartículas están suspendidas en el aire y pueden tener contacto con la piel no protegida (figura 3 ).

Cabe mencionar que independientemente de la vía de exposición, cierta cantidad puede traslocarse a torrente sanguíneo y posteriormente depositarse en diversos órganos. Por otro lado, mediante la vía inhalatoria, los na- 
FIGURA 3. Principales vías de exposición a nanomateriales (NM). La vía oral (amarillo) ocurre mediante el consumo de alimentos, pastas dentales o medicamentos. La vía inhalatoria (azul) ocurre en los ambientes ocupacionales donde se manufacturan los nanomateriales o donde se usan para fabricar productos que los contienen. La vía dérmica (verde) ocurre mediante el uso de productos cosméticos o por exposición ocupacional.

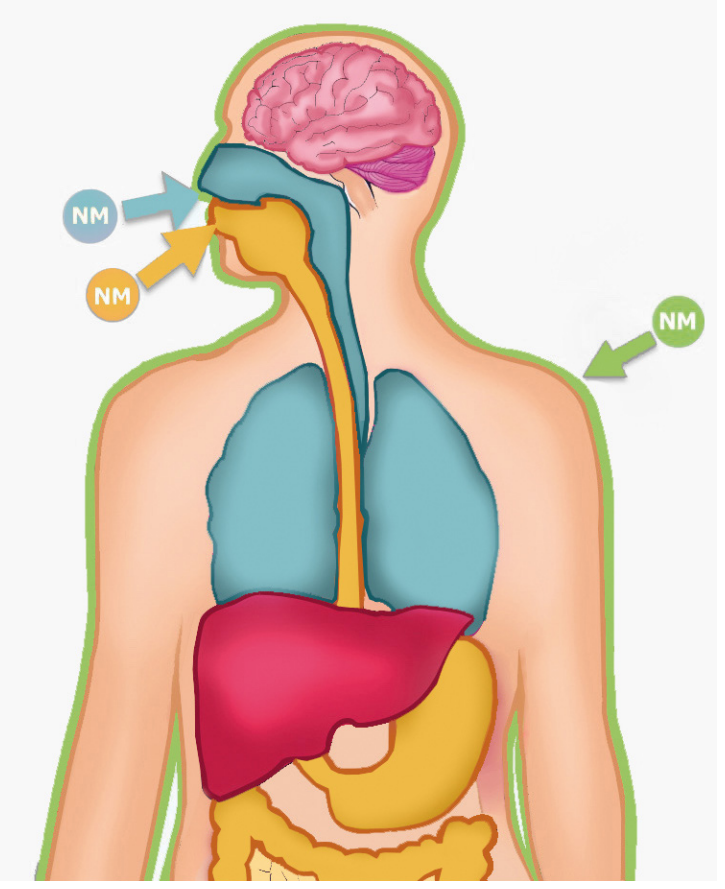

Fuente: Elaboración de las autoras.

nomateriales pueden llegar al cerebro mediante el bulbo olfatorio. Si el órgano en el que se depositan los nanomateriales presenta alguna patología, dicho depósito podría representar un riesgo para exacerbarla. En el caso del cerebro, la acumulación de nanomateriales podría representar un riesgo para el desarrollo de enfermedades neurodegenerativas o agravar también patologías prexistentes.

\section{Aplicaciones de los nanomateriales en sistema nervioso}

\section{Ventajas}

En el campo de la medicina algunos nanomateriales tienen aplicaciones en el sistema nervioso, a tal grado que se han denominado neuronanomateriales, un término bastante reciente. Estas aplicaciones incluyen la liberación de fármacos y genes cubiertos por el nanomaterial en el sistema nervioso y que permitan la llegada al sitio de interés a través de la barrera hematoencefálica (Kaushik et al., 2017). Un ejemplo específico es la tecnología G o G-Techno- 
logy ${ }^{\circledast}$ (to-BBB, Leiden, The Netherlands). Ésta consiste en la administración de fármacos encapsulados dentro un liposoma que tiene en su superficie polietilenglicol. Adicionalmente, el polietilenglicol tiene unido glutatión, el cual es de utilidad porque puede cruzar de la barrera hematoencefálica. Una vez que el liposoma ha cruzado dicha barrera puede liberar el fármaco en el cerebro. Este tipo de fármacos pueden ser utilizados para el tratamiento de enfermedades neurodegenerativas como el Alzheimer, Parkinson o tumores en cerebro, entre otros (Kaushik et al., 2017). Los nanomateriales también se pueden usar para obtener imágenes del cerebro. Un ejemplo son las nanopartículas superparamagnéticas de óxido de hierro, las cuales pueden ser controladas por campos magnéticos para detectar la actividad cerebral o localizar regiones del cerebro si se les acopla anticuerpos específicos (Ohtake et al., 2017). Estas regiones podrían corresponder a zonas en donde haya lesiones por traumatismos o por enfermedades.

\section{Desventajas}

En general, los nanomateriales después de depositarse dentro del cuerpo no son degradados fácilmente y pueden retenerse incluso por varios meses causando alteraciones dentro de las células donde se hayan depositado. Tal es el caso de las nanopartículas de plata y de dióxido de silicio que se acumulan en el cerebro de ratas durante 12 semanas de exposición (Parveen et al., 2014; Wen et al., 2015), además, causan apoptosis y disminución de la viabilidad celular (Hsiao et al., 2017), así como alteraciones de la morfología celular y producción de especies reactivas de oxígeno, respectivamente (Yang et al., 2014). Es por ello que ha surgido la preocupación de si los neuronanomateriales podrían causar neurotoxicidad después de ejercer su efecto, por caso, después de liberar un fármaco, debido a la baja capacidad para ser eliminados del sistema nervioso. Además, la exposición accidental, como por ejemplo cuando se inhalan y pasan del bulbo olfatorio al cerebro, podría causar su acumulación y se desconoce si podrían alterar las funciones de las zonas del cerebro en donde se depositan.

\section{¿Cómo podrían causar daño los neuronanomateriales?}

Después de ser inhalados o consumidos oralmente, los nanomateriales pueden llegar al torrente sanguíneo y entonces atravesar la barrera hematoencefálica, pero también pueden cruzarla mediante el bulbo olfatorio después de ser inhalados (Dumková et al., 2017). De estas formas podrían llegar a sistema nervioso central y depositarse en diferentes zonas del cerebro causando:

- Daño estructural de la barrera hematoencefálica. Al atravesar la barrera hematoencefálica, los nanomateriales pueden causar daño físico dañando las células que componen el sistema nervioso como astrocitos y células endoteliales afectando la capacidad de seleccionar las 
sustancias que entran y salen de la barrera hematoencefálica (Disdier et al., 2015).

- Formación de especies oxidantes que promueven inflamación. Las especies oxidantes son aquellas que derivan del oxígeno y son conocidas como especies reactivas de oxígeno, Ros por sus siglas en inglés. Estas especies se producen en forma fisiológica pero en exceso oxidan biomoléculas como lípidos, carbohidratos y DNA causando daño estructural y funcional en las células en las que se producen. En condiciones severas, la excesiva producción de estas especies puede llevar a la muerte celular (Ze et al., 2014).

- Empeoramiento de enfermedades neurodegenerativas. Es posible que si una persona padece alguna enfermedad neurodegenerativa, la acumulación de nanomateriales pueda acelerar o empeorar enfermedades como Alzheimer, Parkinson y Huntington (Liu et al., 2017), pero también podría aumentar el riesgo de padecer algún accidente cerebrovascular.

\section{Estudios de toxicidad de nanomateriales en el SNC}

Se han realizado estudios en los que se evalúa la toxicidad de diferentes nanomateriales en sistema nervioso central y se ha demostrado en modelos experimentales que, por ejemplo, las nanopartículas de dióxido de titanio causan afectaciones en la respuesta inmune, en la memoria, el aprendizaje, el desarrollo cerebral (Engler-Chiurazzi et al., 2016) y también, cuando se inhalan, neurodegeneración (Win-Shwe y Fujimaki, 2011). Además, estas nanopartículas inducen la activación de la microglía contribuyendo a la vulnerabilidad neuronal a causa de la liberación de inmunomoduladores como citocinas (De Astis et al., 2013).

Cuando las nanopartículas de dióxido de titanio ingresan al cerebro por la vía inhalatoria pueden depositarse en el bulbo olfatorio (Pujalté et al., 2017) y en el hipocampo (Ze et al., 2014). El hipocampo está relacionado principalmente con la enfermedad de Alzheimer.

También se ha visto que las nanopartículas de oro pueden cruzar la barrera hematoencefálica y depositarse en el cerebro. En cerebros de rata, se ha observado que las nanopartículas de oro causan oxidación de lípidos, reducción de los niveles de glutatión peroxidasa, que es una enzima que degrada especies oxidantes como el peróxido de hidrógeno, así como un incremento en los niveles de 8-hidroxideoxiguanosina, un marcador de daño a DNA.

Por su parte, las nanopartículas de dióxido de silicio también pueden cruzar la barrera hematoencefálica y depositarse en diferentes zonas del cerebro, como en el cuerpo estriado (Parveen et al., 2014), el hipocampo, entre otras (Wu et al., 2011). Estas nanopartículas disminuyen la defensa antioxi- 
dante, aumentan la cantidad de Ros y promueven la liberación de citocinas proinflamatorias.

\section{¿Es indudable que causen daño?}

La respuesta es "no". Debido a que la gran mayoría de los estudios se ha realizado con cultivos celulares y con modelos animales experimentales y en algunos casos las concentraciones y dosis usadas son relativamente altas debido a que no existen todavía estudios precisos de qué concentraciones se alcanzan en SNC cuando los seres humanos están expuestos por vía intravenosa o inhalatoria. En este caso, los efectos pueden estar siendo magnificados pero por otro lado, los sistemas de estudio in vivo emplean animales sanos, genéticamente estandarizados y aunque las dosis sean altas, los efectos en el humano podrían ser peores. Por ejemplo, un grupo de animales está expuesto a nanopartículas en concentraciones 10 veces mayores a las reales y los resultados pueden mostrar cierto grado de toxicidad. Sin embargo, el ser humano, puede ser un individuo con predisposición genética a ciertas enfermedades, con sedentarismo, dieta baja en fibra y alta en carbohidratos, ser fumador y dormir pocas horas por día. Todos estos factores, a pesar de estar expuesto dicho ser humano a una dosis baja de nanopartículas, podrían hacerle presentar mayores signos de toxicidad en SNC que los predichos en el estudio con animales en el que se empleó una dosis de nanopartículas10 veces menor.

Es por ello que es indispensable dar seguimiento a las investigaciones que evalúen todos los tipos de nanomateriales que pudieran llegar a sistema nervioso central, ya sea por translocación vía bulbo olfatorio o como resultado de su depósito al usarse en aplicaciones médicas. Los estudios deben responder qué tan seguro es el uso de nanomateriales en aplicaciones médicas, especialmente cuando su uso es prolongando, así como cuál es su contribución en el desarrollo o progresión de enfermedades neurodegenerativas. Por otro lado, deben indicar los posibles daños que ocurrirían en cerebro cuando los nanomateriales sean inhalados o consumidos vía oral, ya que por ambas vías también pueden traslocarse al torrente sanguíneo.

\section{Consideraciones finales}

Hasta el momento existe evidencia de que las nanopartículas pueden cruzar la barrera hematoencefálica ya sea porque llegan al torrente sanguíneo al ser inhaladas mediante el bulbo olfatorio o al ser ingeridas en alimentos o medicamentos, en donde, eventualmente, también pueden alcanzar el torrente sanguíneo. Si se administran en medicamentos vía intravenosa, la concentración que se alcanza podría ser mucho mayor. Existe evidencia derivada de modelos in vitro e in vivo que indica que el depósito de nanomateriales puede causar alteraciones en las células en que se internalizan, especialmente si su 
naturaleza química les impide ser degradadas dentro de la célula. Sin embargo, debe centrarse la atención en el diseño de los estudios evitando la exposición a nanomateriales en concentraciones no realistas, es decir, concentraciones superiores a las que un ser humano podría estar expuesto, ya sea por vía oral, inhalatoria o intravenosa, entre otras vías. Es importante también diseñar estudios que permitan conocer cuáles serían las consecuencias de estar expuesto a nanomateriales no sólo en condiciones de salud, sino en presencia de patologías preexistentes incluyendo enfermedades neurodegenerativas con el objetivo de determinar si la exposición podría exacerbar dicha enfermedad y en qué medida. Los estudios también deben enfocarse en determinar si los nanomateriales usados en medicina para liberación de fármacos y diagnóstico son seguros después de ejercer su efecto benéfico, ya que muchos de los nanomateriales, por ejemplo, óxidos metálicos o de base de oro, no son degradables por las células que los internalizan. Por último, es importante diseñar estudios en poblaciones susceptibles como niños y adultos mayores, en cuyo caso, el depósito de nanomateriales en sistema nervioso central puede tener consecuencias más severas que en adultos sanos pues casi todos los modelos experimentales excluyen justamente la representación de ellos al realizarse con animales adultos.

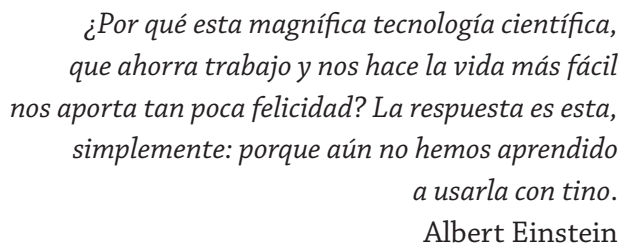

\section{Referencias}

De Astis, S., Corradini, I., Morini, R., Rodighiero, S., Tomasoni, R., Lenardi, C., Verderio, C., Milani, P., Matteoli, M. (2013). Nanostructured $\mathrm{TiO}_{2}$ surfaces promote polarized activation of microglia, but not astrocytes, toward a proinflammatory profile. Nanoscale, 5(22): 10963-10974. https://doi.org/10.1039/c3nr03534d

Disdier, C., Devoy, J., Cosnefroy, A., Chalansonnet, M., Herlin-Boime, N., Brun, E., Lund, A. y Mabondzo, A. (2015). Tissue biodistribution of intravenously administrated titanium dioxide nanoparticles revealed blood-brain barrier clearance and brain inflammation in rat. Particle and Fibre Toxicology, 12: 27. https://doi.org/10.1186/s12989-015-0102-8

Dumková, J., Smutná, T., Vrlíková, L., Le Coustumer, P., Večeřa, Z., Dočekal, B., Mikuška, P., Čapka, L., Fictum, P., Hampl, A. y Buchtová, M. (2017). Subchronic inhalation of lead oxide nanoparticles revealed their broad distribution and tissue-specific subcellular localization in target organs. Part Fibre Toxicology, 14(1): 55. https://doi.org/10.1186/s12989-017-0236-y 
Engler-Chiurazzi, E. B., Stapleton, P. A., Stalnaker, J. J., Ren, X., Hu, H., Nurkiewicz, T. R., McBride, C. R., Yi, J., Engels, K. y Simpkins, J. W. (2016). Impacts of prenatal nanomaterial exposure on male adult Sprague-Dawley rat behavior and cognition. Journal of Toxicology and Environmental Health, Part A, 79(11): 447-452. https://doi.org/10.1080/15287394.2016.1164101

Hsiao, I.L., Hsieh, Y. K., Chuang, C. Y., Wang, C. F. y Huang, Y. J. (2017). Effects of silver nanoparticles on the interactions of neuron- and glia-like cells: Toxicity, uptake mechanisms, and lysosomal tracking. Environmental Toxicology, 32(6): 1742-1753. https://doi.org/10.1002/tox.22397

Kaushik, A., Jayant, R. D., Bhardwaj, V. y Nair, M. (2017). Personalized nanomedicine for CNS diseases. Drug Discovery Today.

https://doi.org/10.1016/j.drudis.2017.11.010

Liu, X., Sui, B. y Sun, J. 2017. Blood-brain barrier dysfunction induced by silica NPs in vitro and in vivo: Involvement of oxidative stress and Rho-kinase/JNK signaling pathways. Biomaterials, 121: 64-82.

https://doi.org/10.1016/j.biomaterials.2017.01.006

Ohtake, M., Umemura, M., Sato, I., Akimoto, T., Oda, K., Nagasako, A., Kim, J. H., Fujita, T., Yokoyama, U., Nakayama, T., Hoshino, Y., Ishiba, M., Tokura, S., Hara, M., Muramoto, T., Yamada, S., Masuda, T., Aoki, I., Takemura, Y., Murata, H., Eguchi, H., Kawahara, N. y Ishikawa, Y. (2017). Hyperthermia and chemotherapy using $\mathrm{Fe}($ Salen) nanoparticles might impact glioblastoma treatment. Scientific Reports, 7: 42783. https://doi.org/10.1038/srep42783

Parveen A, Rizvi S. H. M., Mahdi, F., Tripathi, S., Ahmad, I., Shukla, R. K., Khanna, V. K., Singh, R., Patel, D. K. y Mahdi, A. A. (2014). Silica nanoparticles mediated neuronal cell death in corpus striatum of rat brain: Implication of mitochondrial, endoplasmic reticulum and oxidative stress. Journal of Nanoparticle Research, 16: 2664. https://doi.org/10.1007/s11051-014-2664-z

Pujalté, I., Dieme, D., Haddad, S. y Serventi, A. M., Bouchard. M. (2017). Toxicokinetics of titanium dioxide $\left(\mathrm{TiO}_{2}\right)$ nanoparticles after inhalation in rats. Toxicology Letters, 265: 77-85. https://doi.org/ 10.1016/j.toxlet.2016.11.014

Thiruvengadam, M., Rajakumar, G., Chung, I. (2018). Nanotechnology: Current uses and future applications in the food industry. 3 Biotech, 8: 74.

https://doi.org/10.1007/s13205-018-1104-7

Unión Europea (2011). Disponible en: http://data.europa.eu/eli/reco/2011/696/oj

Wen, R., Yang, X., Hu, L., Sun, C., Zhou, Q. y Jiang, G. (2015). Brain-targeted distribution and high retention of silver by chronic intranasal instillation of silver nanoparticles and ions in Sprague-Dawley rats. Journal of Applied Toxicology, 36(3): 445-53.

Win-Shwe, T. T. y Fujimaki, H. (2011). Nanoparticles and neurotoxicity. International Journal of Molecular Sciences, 12(9): 6267- 6280. https://doi.org/10.3390/ijms12096267

Wu, J., Wang, C., Sun, J. y Xue, Y. (2011). Neurotoxicity of silica nanoparticles: Brain localization and dopaminergic neurons damage pathways. ACS Nano, 5 (6): 4476-4489. https://doi.org/10.1021/nn103530b 
Yang, X., He, C., Li, J., Chen, H., Ma, Q., Sui, X., Tian, S., Ying, M., Zhang, Q., Luo, Y., Zhuang, Z. y Liu, J. (2014). Uptake of silica nanoparticles: neurotoxicity and Alzheimer-like pathology in human SK-N-SH and mouse neuro2a neuroblastoma cells. Toxicology Letters, 229(1): 240-249.

https://doi.org/10.1016/j.toxlet.2014.05.009

Ze, Y., Sheng, L., Zhao. X., Hong, J., Ze, X., Yu, X., Yu, X., Pan, X., Lin, A., Zhao, Y., Zhang, C., Zhou, Q., Wang, L. y Hong, F. (2014). $\mathrm{TiO}_{2}$ Nanoparticles induced hippocampal neuroinflammation in mice. PLoS ONE, 9(3): e92230.

https://doi.org/10.1371/journal.pone.0092230 